\title{
Clinician’s Commentary
}

Inness et al.'s well-designed measurement study ${ }^{1}$ provides convincing evidence about key aspects of the Community Balance and Mobility (CB\&M) Scale's construct validity and responsiveness to change for a rehabilitationcentre-based sample of adults with traumatic brain injury (TBI). The work is strengthened by the use of a set of comparison measures chosen both to reflect the underlying constructs of interest and to span the International Classification of Functioning, Disability and Health (ICF) areas of "body function and structures," "activity," and "participation." 2 Their study encourages clinicians to think beyond standard measures of balance, such as gait assessments and the Berg Balance Scale (BBS), ${ }^{3}$ to achieve a more sensitive clinical assessment of the persistent high-level balance/mobility deficits that individuals with TBI often experience.

Several of the authors of this validation study are members of the development team for the CB\&M, which was created specifically to address this measurement challenge. Their continuing work with this observational assessment reflects the length of time, critical thinking, research action, and funding required to fully validate a new outcome measure. The growing body of wellintegrated evidence on the CB\&M's properties shows the value of this long-term commitment on the part of its developers. To fully demonstrate a measure's psychometric properties and clinical uptake, however, it is also critical that researchers in other settings get involved in validation efforts. The CB\&M is showing promise in this regard. The timing of the release of this paper coincides with the publication of CB\&M validation work by two other Canadian research teams (Knorr et al. ${ }^{4}$ for adults with stroke, and Wright et al. ${ }^{5}$ for children and youth with an acquired brain injury $[\mathrm{ABI}])$. The publication of these papers in three different journals (Physiotherapy Canada, Archives of Physical Medicine and Rehabilitation, and Brain Injury) should help to increase the CB\&M's international profile.

Several important clinical messages arise from this study, both for those who provide physiotherapy to clients with TBI and for managers and decision makers who determine the type and extent of the physiotherapy services supported. Specifically, Inness et al.'s paper provides quantitative evidence that high-level dynamic balance issues observed during performance of advanced motor skills are linked to the individual's ability to move safely and to participate in community-based activities. This connection is exemplified by moderate to strong correlations between the CB\&M and the Community Integration Questionnaire (CIQ) and between the CB\&M and the Activities-specific Balance Confidence (ABC) Scale. While one could argue that these results simply confirm what physiotherapists who specialize in TBI and stroke already "know" from their clinical experience, the empirical support for this knowledge is an essential first step toward supporting physiotherapy interventions that focus on enhancing high-level balance skills. The next step, which is now well set up by the authors' demonstration of the CB\&M's lack of ceiling effect and its high responsiveness to change, is use of the CB\&M in clinical trials that rigorously investigate the actual effectiveness of these physiotherapy interventions.

The presence of strong relationships between the $\mathrm{CB} \& \mathrm{M}$ and the impairment measures studied (e.g., correlations generally $>0.70$ ) does not mean that one measure should be used in place of the other(s), though this might be tempting from the standpoint of assessment efficiency. There is evidence from paediatric rehabilitation that measures that are highly correlated at a given time point may not continue to show a strong association as changes in performance occur. ${ }^{6}$ Taking a hypothetical example from the CB\&M, while step length and the CB\&M are strongly correlated at one time point, opportunities to practise skills in the community may be associated with overall improvements in CB\&M scores even though underlying gait features such as step length may remain unchanged; in this case there could be low change-score correlations between the CB\&M and step length. In future efforts to elucidate the relationships among ICF areas with respect to change, therefore, a core set of outcomes needs to include measures that represent a variety of different constructs. This will provide opportunities to look at change-score associations and predictors of change so as to better understand the interplay of various factors across the ICF.

From the perspective of CB\&M administration and use, Inness et al. note that their research has not yet answered the question of the level of ambulatory skill needed before a patient can be safely and suitably assessed on the CB\&M. Specifically, what is the minimum score on easier balance measures, such the BBS or the timed up-and-go (TUG), ${ }^{7}$ that signals that the patient can safely try the CB\&M tasks? Inness et al. note that their study sample did not use the CB\&M at its lowest scoring extreme, and may thus have missed those patients who are commonly seen for rehabilitation and gait training following TBI. We experienced the same score situation in our CB\&M reliability sample of children with an $\mathrm{ABI}$, and wondered about the minimum Gross Motor Function Measure $(\mathrm{GMFM})^{8}$ score requirements for safe 
performance on the CB\&M that would allow better use of the lower range of CB\&M scores. ${ }^{5}$ An investigation of lower scores was part of the CB\&M study objectives in recent research by Knorr et al. ${ }^{4}$ in adults with stroke. While there was some indication of a floor effect in their sample, it was perhaps less than anticipated, occurring in about $7 \%$ of the sample. Knorr et al. ${ }^{4}$ suggested a BBS score $\geq 40$ or a TUG score $\leq 30$ seconds as the cut-point for safe use of the CB\&M with stroke survivors.

Evidence-informed guidelines such as those provided by Knorr et al. ${ }^{4}$ will be helpful to clinicians in deciding when to begin using the CB\&M with a patient. In order to get an idea of an individual's changes in balance across the improvement continuum, it might be useful for the physiotherapist to consider basic balance skills using the BBS (i.e., pre-CB\&M) and then, when abilities improve and BBS scores near a specified cut-point or reach the BBS ceiling, either employ both the BBS and the CB\&M or transition completely to the CB\&M. Translation of scores across measures, known as cross-calibration, allows transformation of values or substitution of measures and is possible using sophisticated statistical approaches. ${ }^{9,10}$ While this approach holds some promise, it is not something that we currently are accustomed to doing with rehabilitation measures. Lastly, use of Rasch scaling methods ${ }^{8}$ might be a first step toward creating unidimensional interval scales for the CB\&M and BBS. This would permit a look at item difficulty progressions within, and potentially across, these two measures.

From the standpoint of clinical accessibility, it is commendable that the CB\&M, like the BBS, is available for free use, along with its test-track set-up and administration guidelines. ${ }^{1,11}$ From a practical standpoint, learning to use the CB\&M requires use of the online CB\&M manual $^{11}$ to guide the testing process, as well as handson practice in the learning stages to develop fluidity of administration according to the guidelines and accuracy of scoring. The importance of respecting the nuances of CB\&M scoring needs to be stressed, since these are pivotal to the reliability and validity observed by these authors and others. While the inclusion of measurement of aspects of quality of movement within the CB\&M's time- and distance-based response scales adds more complexity to the rater's judgment of performance, reliability work has shown that high interrater reliability can be attained with training and well-operationalized rating guidelines.

Although it safe to say that validation work is never really finished, at this stage we can be satisfied with the scope of the body of work establishing reliability and construct/criterion validity for the CB\&M across areas of the ICF. We also now have a good idea about responsiveness, both from Inness et al. ${ }^{1}$ (i.e., a large effect size) and from similar results in Knorr et al.'s work. ${ }^{4}$ Measurement science is evolving, however, and clinicians' knowledge requirements for measure implementation continue to grow. It is indeed time to move forward with CB\&M to examine other measurement concepts. Where do we go from here? Inness et al. note that one of the next steps will be to establish clinically important change values so that guidelines for interpreting $C B \& M$ change scores can be developed. This is a challenging but critical endeavour that parallels work either underway or called for with respect to most of our other rehabilitation outcome measures. Ideas about how this work might be undertaken are available in an excellent publication by Wells et al. ${ }^{12}$

Finally, the expectation is that there will be an increasing international uptake of this carefully developed and well-validated measure. The CB\&M's suitability to evaluate high-level balance in ABI (adult and paediatric) and stroke has been established, as has its potential to bridge the measurement gap from childhood to adulthood. The latter ability bodes well for the study of longterm outcomes for youth who have a brain injury. As we have seen with the GMFM, it takes the collective work of international clinical and research communities to help an outcome measure reach its full potential. It is hoped that the recent papers on the CB\&M by three different Canadian research groups will be the spark that catches the attention of physiotherapists and rehabilitation teams around the world.

F. Virginia Wright, $P T, P h D$

Clinician Scientist, Bloorview Research Institute and Holland Bloorview Kids Rehabilitation Hospital Assistant Professor (status), Department of Physical Therapy, University of Toronto Toronto, ON vwright@hollandbloorview.ca

\section{REFERENCES}

1. Inness EL, Howe JA, Niechwiej-Szwedo E, Jaglal SB, McIlroy WE, Verrier MC. Measuring balance and mobility after traumatic brain injury: validation of the Community Balance and Mobility Scale (CB\&M). Physiother Can. 2011;63:199-208. doi:10.3138/ptc.2009-45

2. World Health Organization. International classification of functioning, disability and health. Geneva: The Organization; 2001.

3. Berg KO, Wood-Dauphinee SL, Williams JI, Maki B. Measuring balance in the elderly: validation of an instrument. Can J Public Health. 1992;83(Suppl.2):S7-11.

4. Knorr S, Brouwer B, Garland SJ. Validity of the Community Balance and Mobility Scale in community-dwelling persons after stroke. Arch Phys Med Rehabil. 2010;91:890-6. doi:10.1016/j.apmr.2010.02.010

5. Wright V, Ryan J, Brewer K. Reliability of the Community Balance and Mobility Scale (CB\&M) in high-functioning school-aged children and adolescents who have an acquired brain injury. Brain Inj. 2010;24:1585-94. doi:10.3109/02699052.2010.523045

6. Wright V, Rosenbaum P, Goldsmith C, Fehlings D, Law M. How do changes in body functions/structures, activity and participation relate to each other in children with cerebral palsy? Dev Med Child Neurol. 2008;50:283-9. 
7. Podsiadlo D, Richardson S. The timed "up \& go": a test of basic functional mobility for frail elderly persons. J Am Geriatr Soc. 1991;39:142-8.

8. Russell DJ, Avery LM, Rosenbaum PL, Raina PS, Walter SD, Palisano RJ. Improved scaling of the Gross Motor Function Measure for children with cerebral palsy: evidence of reliability and validity. Phys Ther. 2000;80:873-85.

9. Sechrest L, McKnight P, McKnight K. Calibration of measures for psychotherapy outcome studies. Am Psychol. 1996;51:1065-71. doi:10.1037//0003-066X.51.10.1065

10. Parmigiani G, Ashih HW, Samsa GP, Duncan PW, Lai SM, Matchar DB. Cross-calibration of stroke disability measures: Bayesian analysis of longitudinal ordinal categorical data using negative dependence [Internet]. Baltimore (MD): Johns Hopkins University, Depart- ment of Biostatistics Working Papers; 2003 Aug [cited 2010 Nov 1]. Available from: http://www.bepress.com/jhubiostat/paper2.

11. Howe JA, Inness L. Community Balance and Mobility Scale manual, revised [Internet]. Toronto: Toronto Rehab Institute and University of Toronto; 2002 [cited 2010 Nov 4]. Available from: http://www.torontorehab.com/research/documents/ CBM\%20Scale\%20Toronto\%20Rehab\%2008021.pdf.

12. Wells G, Beaton D, Shea B, Boers M, Simon L, Strand V, et al. Minimal clinically important differences: review of methods. J Rheumatol. 2001;28:406-12.

D0I:10.3138/physio.63.2.209 